\title{
Self-assembled nanostructured photosensitizer with aggregation-induced emission for enhanced photodynamic anticancer therapy
}

\author{
Wenkun $\mathrm{Han}^{1}$, Song Zhang ${ }^{1}$, Rong Deng ${ }^{2}$, Yangyang Du ${ }^{2}$, Jingyu Qian ${ }^{1}$, Xiaohua Zheng ${ }^{3}$, Bin $\mathrm{Xu}^{1}$,
} Zhigang Xie ${ }^{3}$, Fei Yan $^{2^{*}}$ and Wenjing Tian ${ }^{1 *}$

\begin{abstract}
Three nanostructured photosensitizers with aggregation-induced emission (AIE) characteristics based on 2,3-bis(4'-(diphenylamino)-[1,1'-biphenyl]-4-yl) fumaronitrile (BDBF) were prepared for image-guided photodynamic therapy (PDT). BDBF was encapsulated with Pluronic F-127 (F127) to form usual spherical nanoparticles (F127@BDBF NPs) with a red fluorescence emission and $\mathbf{9 . 8 \%}$ fluorescence quantum yield (FQY). Moreover, BDBF self-assembled into nanorods (BDBF NRs) in water. Compared with F127@BDBF NPs, BDBF NRs exhibited stronger orange fluorescence with a higher FQY of $23.3 \%$ and similar singlet oxygen $\left({ }^{1} \mathrm{O}_{2}\right)$ generation capability. BDBF NRs were further modified with F127 to form BDBF@F127 NRs with the same ${ }^{1} \mathrm{O}_{2}$ generation ability as BDBF NRs. The three nanostructures exhibited a higher ${ }^{1} \mathrm{O}_{2}$ production capacity than BDBF molecule in dissolved state and favorable stability in an aqueous solution as well as under physiological condition. In vitro photocytotoxicity experiments indicated that the three nanostructures inhibited tumor cell proliferation effectively. Therefore, to construct eligible nanostructures with a high FQY and ${ }^{1} \mathrm{O}_{2}$ generation ability, simple self-assembly can serve as a valuable method to prepare photosensitizers with enhanced PDT.
\end{abstract}

Keywords: aggregation-induced emission, photosensitizer, nanorods, photodynamic therapy

\section{INTRODUCTION}

Photodynamic therapy (PDT) is an important cancer therapy strategy and has attracted much attention because of its controllable noninvasive treatment modality and high spatiotemporal precision [1-6]. PDT has three key factors: photosensitizer (PS), light, and endogenous oxygen [7-10]. In general, the three elements are harmless for tissues; however, when PS is exposed to a specific light source, the virulent reactive oxygen species (ROS), e.g., singlet oxygen $\left({ }^{1} \mathrm{O}_{2}\right)$, are generated, causing cell apoptosis and further inhibiting tumor growth [11-17]. As one of the important factors of PDT, multitudinous PSs, such as porphyrin derivatives, boron-dipyrromethene (BODIPY) derivatives, and phthalocyanine derivatives, have been designed and synthesized [18-25]. Although PSs have been extensively improved, they still suffer from drawbacks such as dark toxicity and inferior photostability under complex physiological conditions and highly ROS environments [8,26-30]. Most conventional PSs often suffer from aggregation-caused fluorescence quenching (ACQ) and sharp reduction in ROS generation in the aggregate state owing to intrinsic rigid planar and hydrophobic structures. This limits the application of PDT to some extent [31-34]. In this regard, it is essential to develop new types of PSs with high fluorescence emission and efficient ${ }^{1} \mathrm{O}_{2}$ generation capability for image-guided PDT.

Tang's group [25,35-42] reported a series of fluorogens with aggregation-induced emission (AIEgens) with bright enhanced emission in the aggregate state and nonemission in the dissolved state because of the restriction

\footnotetext{
${ }^{1}$ State Key Laboratory of Supramolecular Structure and Materials, College of Chemistry, Jilin University, Changchun 130012, China

${ }^{2}$ State Key Laboratory of Inorganic Synthesis and Preparative Chemistry, International Joint Research Laboratory of Nano-Micro Architecture Chemistry (NMAC), International Research Center for Chemistry-Medicine Joint Innovation, College of Chemistry, Jilin University, Changchun 130012, China

${ }^{3}$ State Key Laboratory of Polymer Physics and Chemistry, Changchun Institute of Applied Chemistry, Chinese Academy of Sciences, Changchun 130022, China

* Corresponding authors (emails: wjtian@jlu.edu.cn (Tian W); feiyan@jlu.edu.cn (Yan F))
} 
of intramolecular motion (RIM). The inherent and distinct properties of AIEgens provide a potential opportunity to explore a new class of PSs to overcome the aforementioned obstacles. PSs with AIE characteristic (AIEgen PSs) often show bright enhanced fluorescence emission as well as retain the ${ }^{1} \mathrm{O}_{2}$ generation capacity in the aggregate state because of the low nonradiative decay and enhanced fluorescence in the solid or aggregate state of AIE molecules, making AIE PSs a superb possibility for image-guided PDT [37,43-48]. An efficient intersystem crossing (ISC) rate $\left(K_{\text {ISC }}\right)$ from the lowest excited state $\left(\mathrm{S}_{1}\right)$ to the lowest triplet state $\left(\mathrm{T}_{1}\right)$ increased the ${ }^{1} \mathrm{O}_{2}$ production ability of PS, and large spin-orbit coupling (SOC) as well as a small $\Delta E_{\mathrm{ST}}$ (the energy gap between singlet and triplet states) could enhance $K_{\text {ISC }}$ [49-52]. To improve the photosensitization of AIEgen PSs, strong electron accepting and donating groups were introduced on different sides of AIEgens' skeleton to construct donor-acceptor (D-A), donor-acceptor-donor (D-A-D), or acceptor-donor-acceptor (A-D-A) systems for accurate tuning of the highest-energy occupied molecular orbital (HOMO) and lowest-energy unoccupied molecular orbital (LUMO) to minimize the $\Delta E_{\mathrm{ST}}$ [11,37-39,53-55]. Another way to minimize $\Delta E_{\mathrm{ST}}$ is to insert conjugated moieties as a linker between donor and acceptor moieties to lengthen the HOMO-LUMO space for efficient ${ }^{1} \mathrm{O}_{2}$ production capability of PSs. The photosensitization capability of AIEgen PSs could also be enhanced by introducing long conjugated skeletons and D-A systems into AIEgen PSs using polymerization strategies [56-60]. Based on the above principles and strategies, many AIEgen PSs with desired chemical structures have been designed and synthesized with good ${ }^{1} \mathrm{O}_{2}$ production capability and high fluorescence quantum yield (FQY) for image-guided PDT applications [61-66]. In addition to molecular design, the fluorescent properties and ${ }^{1} \mathrm{O}_{2}$ generation capabilities of AIEgen PSs could also be improved by constructing different nanostructures. For example, Liu et al. [67] fabricated two AIE dots using AIEgen PS 2,6-bis(4-(2,2-bis(4-methoxyphenyl)-1-phenylvinyl)phenyl)anthracene-9,10-dione (BTPEAQ) with strong intramolecular charge transfer (ICT) characteristics as the core and 1,2-distearoyl-sn-glycero-3-phosphoethanolamine- $N$-[maleimide(polyethyleneglycol)2000] (DSPE-PEG-Mal) as the matrix or solid silica as the shell for image-guided photodynamic cancer cell ablation. Different encapsulation matrices exhibited different effects on the ICT property and molecular aggregation of BTPEAQ, leading to different emission characteristics and ${ }^{1} \mathrm{O}_{2}$ quantum yields. Recently, Liu et al. [68] prepared nanocrystals based on AIEgen PS 2-((4-(2,2-bis(4-methoxyphenyl)-1-phenylvinyl)phenyl)(thiophen-2-yl)methylene)malononitrile (TPETP) with donor- $\pi$-acceptor structure using a nanocrystallization strategy for therapeutic applications. Compared with the nanoaggregates of TPETP, TPETP in nanocrystals had a more compact and ordered crystal structure to suppress the intramolecular motion, resulting in a higher ${ }^{1} \mathrm{O}_{2}$ generation capability and FQY simultaneously. Furthermore, nanostructures with diverse sizes, versatile morphologies, and various surface properties have different advantages in bioimaging and cancer therapy [69]. Liu's group [70] developed a series of AIEgen-based fluorescent polymeric nanorods by controlling the ultrasound sonication times to impel the nanodot-to-nanorod transition for enhanced cancer targeting and imaging. The AIE nanorods showed more efficient cellular uptake, higher accumulation, and deeper penetration compared with AIE nanodots. Recently, Dong et al. [25,71-73] also prepared several nanostructures with different morphologies based on diketopyrrolopyrrole derivatives using simple reprecipitation methods for photoacoustic imaging-guided photothermal therapy. Xie and Xu et al. [74] obtained stable AIE nanoparticles (NPs) with different rod-like or spherical morphologies by assembling poly(ethylene glycol)-block-poly(L-lactic acid) $\left(\mathrm{PEG}_{5 \mathrm{k}}-\mathrm{PLA}_{10 \mathrm{k}}\right)$ or poly (ethylene glycol)-block-poly(caprolactone) ( $\mathrm{PEG}_{5 \mathrm{k}}{ }^{-}$ $\mathrm{PCL}_{10 \mathrm{k}}$ ) with 9,10-distyrylanthracene (DSA) for noninvasive long-term imaging. Rod-like NPs could be easily internalized than spherical particles, resulting in a better imaging effect.

As one of the classical AIEgens, 2,3-bis(4'-(diphenylamino)-[1,1'-biphenyl]-4-yl) fumaronitrile (BDBF) has been widely utilized in organic light-emitting diodes (OLEDs), long-term cellular imaging, and three-photon fluorescence (3PF) imaging due to its unique structure and excellent optical properties [75-79]. Because of its unique D-A-D structure and good HOMO-LUMO separation $\left(\Delta E_{\mathrm{ST}}=0.37 \mathrm{eV}\right), \mathrm{BDBF}$ could also be used as an AIEgen PS. Tang et al. [58] enhanced the photosensitization efficiency of BDBF PSs by 5.8 times by polymerizing $\mathrm{BDBF}$ into a polymer to extend the molecular conjugated structure. Furthermore, Liu et al. [57] reported a crosslinked porous conjugated polymer PS based on $\mathrm{BDBF}$ with efficient ${ }^{1} \mathrm{O}_{2}$ production and good activity in sunlight-induced green photooxidation. They investigated the ${ }^{1} \mathrm{O}_{2}$ generation efficiencies of polymer PS in detail using time-dependent density functional theory (TD-DFT) and found that the polymerization decreased the energy levels of $\mathrm{BDBF}$ and provided more energy 
transition channels beneficial to facilitating ${ }^{1} \mathrm{O}_{2}$ generation. However, the FQY of polymerized BDBF is often reduced with the increase in ${ }^{1} \mathrm{O}_{2}$ generation efficiency.

Herein, three biocompatible nanostructures based on BDBF were prepared using reprecipitation method (Scheme 1). BDBF self-assembled into nanorods (BDBF NRs) in water without auxiliary. The obtained BDBF NRs were further modified with F127 to fabricate BDBF@F127 NRs with the same morphology as BDBF NRs. Moreover, BDBF and F127 were introduced simultaneously slowly into water to fabricate F127@BDBF NPs. BDBF NRs and BDBF@F127 NRs showed identical fluorescence emission peak at $583 \mathrm{~nm}$, similar FQYs and ${ }^{1} \mathrm{O}_{2}$ production capabilities, whereas F127@BDBF NPs showed different fluorescence emission at $664 \mathrm{~nm}$ with a comparative low FQY, but similar ${ }^{1} \mathrm{O}_{2}$ generation capability compared with BDBF NRs and BDBF@F127 NRs. The three nanostructures could be utilized for bioimaging and killing tumor effectively, indicating that they could be endocytosed by cancer cells and applied for anticancer PDT. The different self-assembly structures of BDBF provided a promising method to fabricate the desired morphologies with high luminescence performance and efficient photosensitization for image-guided PDT.

\section{EXPERIMENTAL SECTION}

\section{Materials}

Sodium methoxide, 4-(diphenylamino)phenylboronic acid, and tetrakis(triphenyl phosphine) palladium $(0)$ were purchased from Shanghai Energy Chemical. 4-Bromophenylacetonitrile was purchased from J\&K Scientific Co., Ltd. Sodium, iodine, diethyl ether, toluene, methanol, $\mathrm{N}, \mathrm{N}$-dimethylformamide (DMF), dichloromethane (DCM), and petroleum ether were purchased from Sinopharm Chemical Reagent Co., Ltd. Diethyl ether was dried by distillation using sodium as the drying agent and benzophenone as the indicator. F127 was purchased from Sigma Aldrich. Fetal bovine serum (FBS) was purchased from Hyclone. Phosphate buffer saline (PBS), Hoechst 33258, Roswell Park Memorial Institute-1640 (RPMI1640) medium, and penicillin-streptomycin solution were purchased from Solarbio Science \& Technology Co., Ltd. Cell Counting Kit-8 (CCK-8) was purchased from Dojindo Molecular Technologies. Distilled water was obtained from a Milli-Q Biocel (Millipore Corporation, Breford, USA) water purification system $(18.25 \mathrm{M} \Omega \mathrm{cm}$ resistivity). HeLa and A549 cell lines were provided by American Type Culture Collection (ATCC). 2',7'-dichlorofluorescein diacetate (DCFH-DA) and Trypan blue

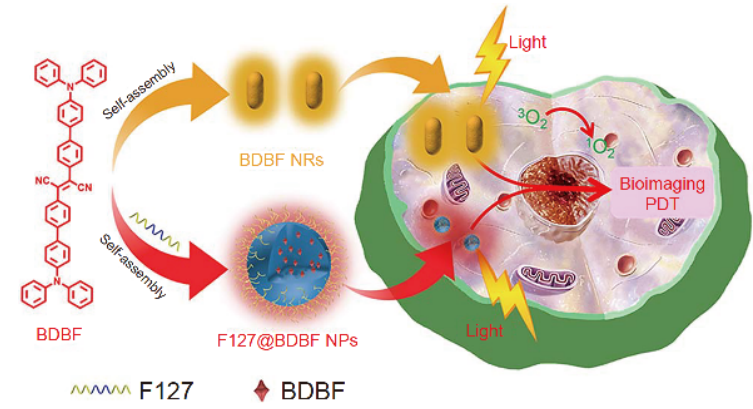

Scheme 1 Schematic of BDBF NRs and F127@BDBF NPs AIEgen PSs nanostructures for image-guided PDT.

were purchased from Shanghai Beyotime Biotechnology Co., Ltd. A cell viability (live dead cell staining) assay kit was purchased from Yeasen Biotech Co., Ltd. Lysotracker Blue and Annexin V-FITC apoptosis detection kit were purchased from Jiangsu KeyGEN Biotechnology Co., Ltd.

\section{Preparation of BDBF}

Fig. S1 shows the synthesis route and chemical structure of BDBF. BDBF was prepared using a Suzuki coupling reaction with a high yield following the literature [75]. The chemical structures and molecular weights of BDBF and the precursors were characterized using proton nuclear magnetic resonance $\left({ }^{1} \mathrm{H}\right.$ NMR) and matrix-assisted laser desorption/ionization time-of-flight mass spectrometry (MALDI-TOF MS) (Figs S2 and S3).

\section{Fabrication of BDBF NRs, BDBF@F127 NRs, and F127@BDBF NPs}

All nanostructures were prepared using the nanoprecipitation method. The BDBF (2 mg) was dissolved in DMF $(3 \mathrm{~mL})$ under sonication for $5 \mathrm{~min}$. After the BDBF stock solution was slowly added into deionized water $(8 \mathrm{~mL})$ under continuous stirring, the mixture was dialyzed against water to remove the remaining DMF, affording BDBF NRs. Then, $10 \mathrm{mg}$ F127 was completely dissolved in deionized water under ultrasonication. Next, the mixture was injected into the prepared BDBF NRs under stirring for $2 \mathrm{~h}$ to obtain BDBF@F127 NRs. Similarly, $2 \mathrm{~mL}$ DMF containing $10 \mathrm{mg}$ F127 and $2 \mathrm{mg}$ BDBF was slowly injected into $10 \mathrm{~mL}$ deionized water under steady stirring. After the stock solution was fully added, the residual DMF was removed by dialysis in deionized water for 24 h. F127@BDBF NPs were obtained without further treatment. The standard calibration curve technique was used to calibrate the concentration of BDBF in BDBF NRs, BDBF@F127 NRs, and F127@BDBF NPs by mea- 
suring the main absorbance of BDBF at $448 \mathrm{~nm}$ by UVvis absorption spectroscopy in 4:1 $v / v \mathrm{DMF} / \mathrm{H}_{2} \mathrm{O}$ mixture (Fig. S4).

\section{Characterization}

${ }^{1} \mathrm{H}$ NMR spectra in $\mathrm{CDCl}_{3}$ at room temperature were obtained using a Bruker AVANCEIII500 NMR instrument. Dynamic light scattering (DLS) measurements were recorded using a Malvern Zeta-sizer Nano instrument at $25^{\circ} \mathrm{C}$, and the scattering angle was fixed at $90^{\circ}$. Transmission electron microscopy (TEM) was carried out using a JEM-2100F electron microscope. MALDI-TOF mass spectra (MS) were recorded using a Bruker/Auto Reflex III mass spectrometer (Bremen, Germany). Fluorescence spectra were measured using a Shimadzu RF-5301 PC spectrometer. UV-vis absorption spectra were measured using a Shimadzu UV-2550 spectrophotometer. Flow cytometry was carried out using an Amnis FlowSight Imaging Flow Cytometer (Merck Millipore, USA). An Olympus FV1000 confocal laser scanning microscope was used to obtain confocal laser scanning microscopy (CLSM) images.

\section{RESULTS AND DISCUSSION}

DLS and TEM analyses were carried out to characterize the size distribution and morphology of nanostructures, respectively. DLS was conducted to measure the average diameter, polydispersity index (PDI), and zeta potential of BDBF NRs, BDBF@F127 NRs, and F127@BDBF NPs.
As shown in Fig. 1 and Table 1, BDBF NRs showed an average hydrodynamic diameter of $179.2 \mathrm{~nm}$ corresponding to a unimodal size distribution $(\mathrm{PDI}=0.084)$. The average size and PDI of BDBF@F127 NRs were $207.0 \mathrm{~nm}$ and 0.097, whereas F127@BDBF NPs showed a good size dispersion $(\mathrm{PDI}=0.216)$ in an aqueous solution with a relatively smaller size of $72.6 \mathrm{~nm}$. The zeta potentials of BDBF NRs, BDBF@F127 NRs, and F127@BDBF NPs were $-44.8,-20.4$, and $-7.69 \mathrm{mV}$, respectively, indicating good stability and dispersibility of these nanostructures in water. The reduction in zeta potential for BDBF@F127 NRs compared with that for BDBF NRs showed that BDBF NRs could be further encapsulated with F127 based on electrostatic interaction. Furthermore, negligible changes in the size and PDI of these nanostructures for 10 days were observed, indicating good stability in an aqueous solution. Besides, all the nanostructures maintained their diameter and PDI for $24 \mathrm{~h}$ in PBS (pH 7.4) containing $10 \% \mathrm{FBS}$ at $37^{\circ} \mathrm{C}$ (Fig. S5) as well as in DMEM and RPMI-1640 with $10 \%$ FBS (Fig. S6), indicating the good stability in physiological environment. Importantly, the morphology of BDBF NRs determined by TEM showed that BDBF could selfassemble into nanorods without F127 auxiliary, whereas F127@BDBF NPs showed dispersed spherical nanoparticles. The morphology of BDBF@F127 NRs characterized by TEM was also nanorods, similar to BDBF NRs, indicating that F127 did not disturb the morphology of BDBF NRs.
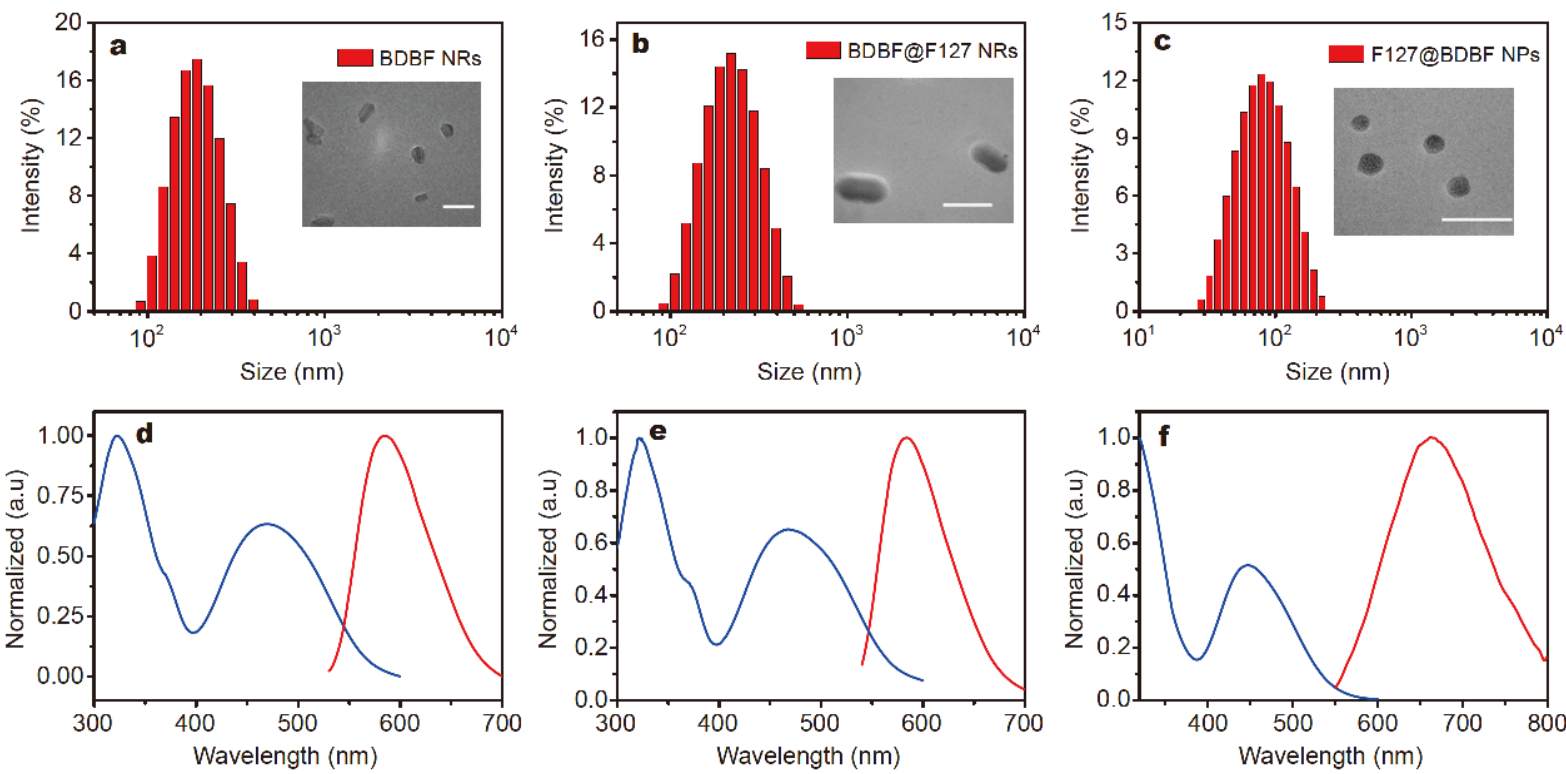

Figure 1 Average sizes of (a) BDBF NRs, (b) BDBF@F127 NRs, and (c) F127@BDBF NPs detected by DLS (inset: TEM images of the corresponding NPs, scale bar: $200 \mathrm{~nm}$ ). Normalized absorbance and fluorescence spectra of (d) BDBF NRs, (e) BDBF@F127 NRs, and (f) F127@BDBF NPs. 
Table 1 Average size, zeta potential, PDI, and FQY of three nanostructures

\begin{tabular}{cccc}
\hline & BDBF NRs & BDBF@F127 NRs & F127@BDBF NPs \\
\hline Size $(\mathrm{nm})$ & 179.2 & 207.0 & 72.6 \\
Zeta $(\mathrm{mV})$ & -44.8 & -20.4 & -7.69 \\
PDI & 0.084 & 0.097 & 0.216 \\
FDY (\%) & 23.3 & 21.9 & 9.8 \\
$\tau_{\text {ave }}(\mathrm{ns})$ & 3.67 & 2.89 & 4.75 \\
\hline
\end{tabular}

Molecular dynamics (MD) simulation was used to further evaluate the self-assembly behavior of BDBF in water. Fig. S7a shows that BDBF was randomly scattered in water in the initial state. With the increase in simulation time, the BDBF molecules constantly accumulated, and completely aggregated after $30 \mathrm{~ns}$, indicating that $\mathrm{BDBF}$ underwent stable aggregation in water. The small aggregates combined to form large agglomerates. From the conformation of BDBF in final aggregates (Fig. S7e), ordered arrangements of $\mathrm{BDBF}$ might contribute to the inhibition of molecular rotations and rigidification of molecular conformation to reduce the possible energy loss by nonradiative rotational relaxation pathway. This might result in a high FQY of BDBF NRs.

The fluorescence spectra of BDBF in water/DMF mixtures with different water fractions $\left(f_{\mathrm{w}}\right)$ were evaluated. As shown in Fig. 2, BDBF showed slight fluorescence in pure DMF, whereas a significant enhanced photoluminescence (PL) intensity at $585 \mathrm{~nm}$ was observed with the change in $f_{\mathrm{w}}$ from $10 \%$ to $30 \%$. The remarkable increase in PL intensity at $30 \% f_{\mathrm{w}}$ confirms the AIE property of BDBF (Fig. S8). When the water fraction became larger than $30 \%$, a clear redshift was observed in the PL spectrum accompanied with a major decrease in the PL intensity due to remarkable ICT of BDBF in more polar environment. According to the literature, DFT calculations of BDBF showed that the HOMO of BDBF was mainly located on the TPA, whereas the LUMO of BDBF was mostly distributed on the central fumaronitrile core. The separation of HOMO and LUMO energy levels of BDBF indicated that BDBF had ICT characteristic. This led to a red shift in the emission maximum, and the emission intensity decreased with increasing solvent polarity $[57,75]$. Moreover, the $\Delta E_{\mathrm{ST}}(0.37 \mathrm{eV})$ of BDBF was small, indicating that BDBF could be used as a PS with significant luminescence emission. The UV-vis absorption and fluorescence spectra of BDBF NRs, BDBF@F127 NRs, and F127@BDBF NPs in aqueous solution as well as their FQYs were further evaluated. As shown in Table 1

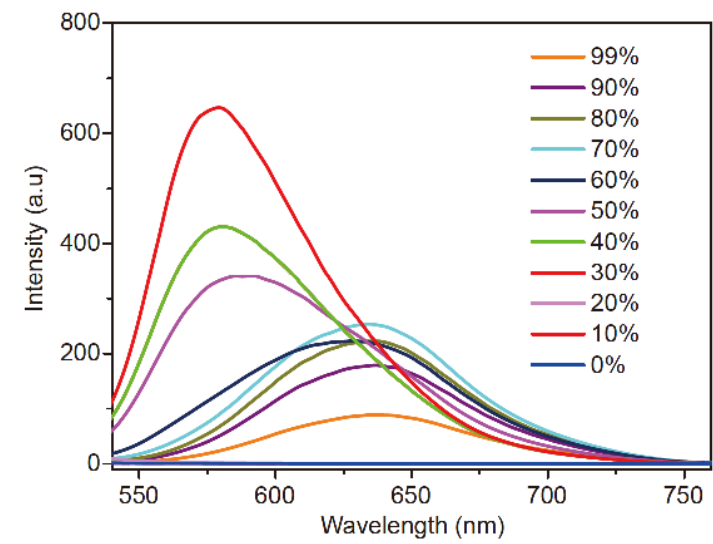

Figure 2 AIE properties of BDBF in DMF with different water fractions.

and Fig. 1, BDBF NRs showed the main absorption peaks at 322 and $468 \mathrm{~nm}$ with an emission maximum at $583 \mathrm{~nm}$ and a FQY of 23.3\%, whereas BDBF@F127 NRs showed similar absorption and emission spectra as well as QY (21.9\%) with BDBF NRs, indicating that F127 did not affect the optical properties of BDBF NRs (Fig. S9). In contrast, compared with BDBF NRs and BDBF@F127 NRs, the absorbance spectra of F127@BDBF NRs exhibited a blue shift to $458 \mathrm{~nm}$, and the emission maximum of F127@BDBF NPs showed a clear bathochromic shift from 583 to $664 \mathrm{~nm}$ with a FQY of $9.8 \%$. Because of different aggregate formation between BDBF NRs and F127@BDBF NPs, the absorbance and emission spectra of BDBF NRs and F127@BDBF NPs were different. The BDBF NRs might have more suppressed intramolecular motion with the more ordered and compact structure, resulting in an increase in brightness as compared with their amorphous nanoaggregates (F127@BDBF NPs). As shown in Fig. S10, the photographs of these nanostructures in water were recorded under daylight and $365 \mathrm{~nm}$ light irradiation. BDBF NRs or BDBF@F127 NRs exhibited bright orange fluorescence emission, whereas F127@BDBF NPs exhibited red fluorescence emission. The time-resolved fluorescence spectra are shown in Fig. S11, and the multiexponential fluorescence decay of the three nanostructures is simulated with average lifetimes of $3.67,2.89$, and 4.75 ns (Table 1) for BDBF NRs, BDBF@F127 NRs, and F127@BDBF NPs, respectively.

Next, indocyanine green (ICG), which quickly decomposed upon interaction with ${ }^{1} \mathrm{O}_{2}$ accompanying decreased absorbance at $790 \mathrm{~nm}[80]$, was used to determine the ${ }^{1} \mathrm{O}_{2}$ generation capability of BDBF NRs, BDBF@F127 NRs, and F127@BDBF NPs upon irradiation by time-dependent UV-vis absorption spectroscopy. As shown in Fig. 3, 

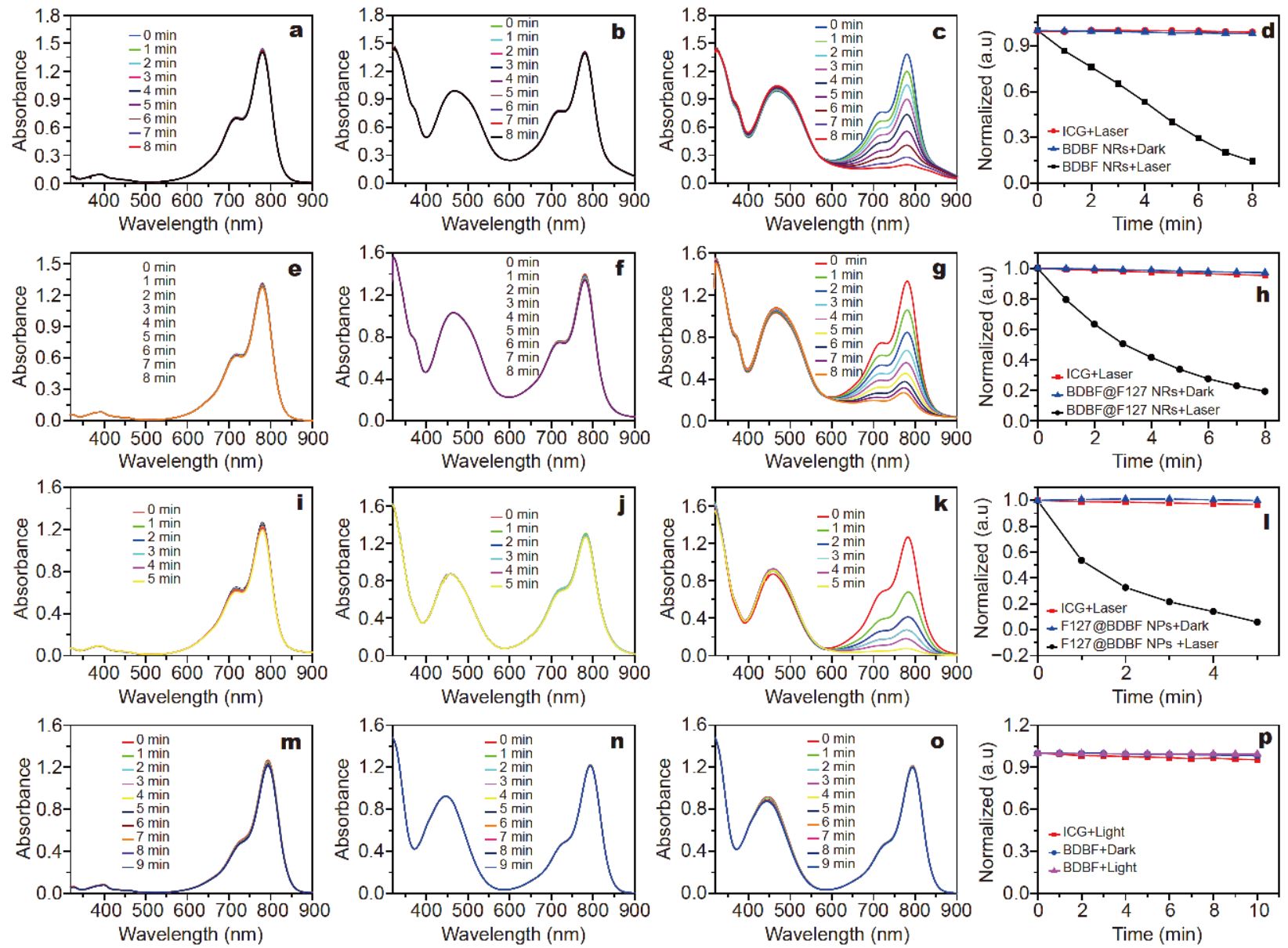

Figure 3 Photostability of ICG in water (a, e, i) and in DMF $(\mathrm{m})$ under light irradiation in the absence of any PS; the absorbance of ICG with the same concentrations of BDBF NRs (b), BDBF@F127 NRs (f), F127@BDBF NPs (j), and BDBF in DMF (n) without light irradiation (BDBF: $30 \mu \mathrm{mol} \mathrm{L}{ }^{-1}$ ); UV-vis absorption spectra changes in the ${ }^{1} \mathrm{O}_{2}$ indicator ICG mixed with BDBF NRs (c), BDBF@F127 NRs (g), F127@BDBF NPs (k), and $\mathrm{BDBF}$ in DMF (o) for different durations of light irradiation; the quantification of ${ }^{1} \mathrm{O}_{2}$ generation capability with the decrease in absorbance at $790 \mathrm{~nm}$ for BDBF NRs (d), BDBF@F127 NRs (h), F127@BDBF NPs (l), and BDBF in DMF (p) under light irradiation $\left(12 \mathrm{~mW} \mathrm{~cm}^{-2}\right)$.

the possibility of photobleaching of ICG by specific light source was eliminated. Negligible decreases were observed at the maximum absorption band of ICG $(790 \mathrm{~nm})$ when ICG was irradiated in water with an LED lamp $\left(450 \mathrm{~nm}, 12 \mathrm{~mW} \mathrm{~cm}^{-2}\right.$ ) for $8 \mathrm{~min}$. However, the maximum absorption of ICG decreased rapidly with light irradiation upon BDBF NRs, BDBF@F127 NRs, and F127@BDBF NPs solutions, indicating the effective generation of ${ }^{1} \mathrm{O}_{2}$ for these nanostructures. Interestingly, the measurements showed BDBF NRs and BDBF@F127 NRs had the similar ${ }^{1} \mathrm{O}_{2}$ production capabilities, indicating that $\mathrm{F} 127$ would not affect the generation of ${ }^{1} \mathrm{O}_{2}$ of BDBF NRs. Compared with BDBF NRs and BDBF@F127 NRs, F127@BDBF NPs also exhibited similar ${ }^{1} \mathrm{O}_{2}$ generation capability, indicating that the distinguishing preparation methods do not disturb the photosensitive effect of
BDBF. Furthermore, the ${ }^{1} \mathrm{O}_{2}$ generation capability of BDBF in DMF was evaluated. Negligible decrease in the absorption of ICG at $780 \mathrm{~nm}$ was observed upon light irradiation when $\mathrm{BDBF}$ was dissolved in DMF, indicating the poor ${ }^{1} \mathrm{O}_{2}$ generation capability of BDBF in DMF, as well as the enhanced ${ }^{1} \mathrm{O}_{2}$ generation in the aggregate state (Fig. S12). Moreover, BDBF NRs, BDBF@F127 NRs, and F127@BDBF NPs simultaneously maintained their photostability with light irradiation.

To evaluate the cellular uptake of BDBF NRs, BDBF@F127 NRs, and F127@BDBF NPs, cellular internalization was performed by CLSM using A549 cells. After incubating A549 cells with BDBF NRs, BDBF@F127 NRs, and F127@BDBF NPs (BDBF: $5 \mu \mathrm{mol} \mathrm{L}^{-1}$ ), the cell nuclei were stained with Hoechst 33258 for $5 \mathrm{~min}$. As shown in Fig. 4, fluorescent signals of BDBF NRs, 

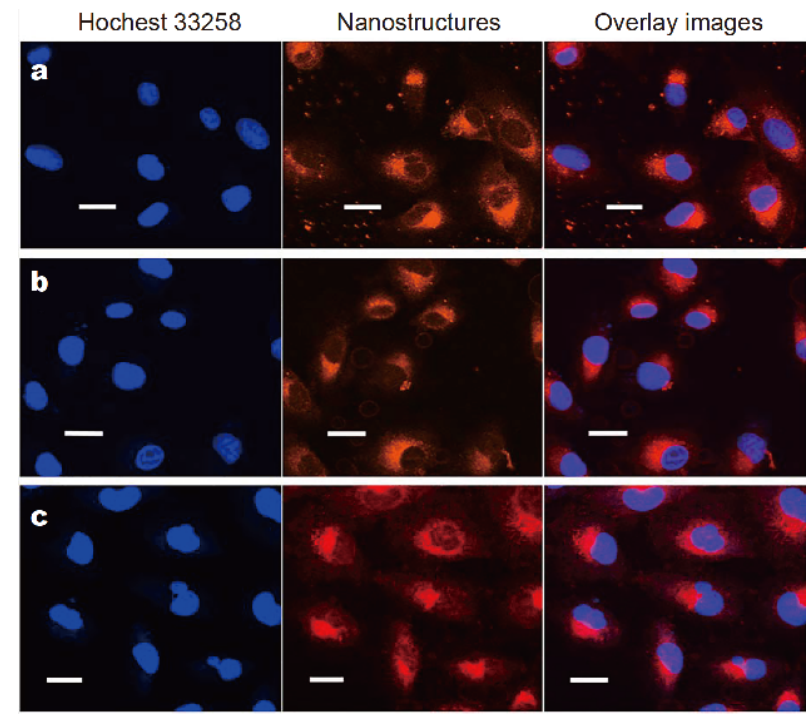

Figure 4 CLSM of A549 cells incubated with BDBF NRs, BDBF@F127 NRs, and F127@BDBF NPs for $4 \mathrm{~h}$ at $37^{\circ} \mathrm{C}$ (BDBF: $5 \mu \mathrm{mol} \mathrm{L}{ }^{-1}$ ). For each panel, the images from left to right show the cell nuclei stained with Hoechst 33258 (blue), BDBF NRs (a), BDBF@F127 NRs (b), and F127@BDBF NPs (c) fluorescence in cells (orange or red), and overlays of both images. Scale bar: $20 \mu \mathrm{m}$.

BDBF@F127 NRs, and F127@BDBF NPs only appeared in the cytoplasm, and A549 cells exhibited red fluorescence, indicating that all nanostructures could be well endocytosed by A549 cells. Cellular uptake experiments were also performed for HeLa cells by CLSM. The confocal images of HeLa cells after incubation with BDBF NRs, BDBF@F127 NRs, and F127@BDBF NPs for $4 \mathrm{~h}$ are shown in Fig. S13. The HeLa cells also exhibited favorable endocytosis towards these nanostructures. The colocalization of endosome (blue) with nanostructures (orange or red) in A549 cells produced a purple fluorescence in the merged images (Fig. S14), indicating that these nanostructures were mainly located within the endosome. The results indicate that these nanostructures can be internalized effectively by cancer cells. The cellular uptake of these nanostructures was further evaluated and quantified using flow cytometry assay. As shown in Fig. S15, the average fluorescence intensities of various nanostructures in A549 cells gradually increased by increasing the incubation time from 0 to $6 \mathrm{~h}$, indicating that all nanostructures were gradually endocytosed with a timedependent behavior.

To evaluate the intracellular ROS generation capabilities of BDBF NRs, BDBF@F127 NRs, and F127@BDBF NPs under appropriate light irradiation, DCFH-DA was used as the scavenger, which could be decomposed into dichlorofluorescein (DCF) under hydrolysis and ROS conditions with bright green fluorescence emission. After A549 cells were incubated with these nanostructures for $6 \mathrm{~h}$, A549 cells irradiated using an LED lamp exhibited stronger green fluorescence than the cells without light irradiation (Fig. S16). Moreover, A549 cells pretreated with F127@BDBF NPs showed similar green fluorescence emission in contrast with those cultivated with BDBF NRs and BDBF@F127 NRs, indicating that all nanostructures exhibited efficient ${ }^{1} \mathrm{O}_{2}$ generation in vitro.

The dark toxicities and phototoxicities of these nanostructures with different concentrations (BDBF: 0-60 $\mu \mathrm{mol} \mathrm{L}^{-1}$ ) against A549 cells were carefully evaluated by CCK-8 assays. As shown in Fig. 5, BDBF NRs, BDBF@F127 NRs, and F127@BDBF NPs showed slight dark toxicities against A549 cells even at a BDBF concentration of $40 \mu \mathrm{mol} \mathrm{L}^{-1}$. When A549 cells were incubated with these nanostructures for $6 \mathrm{~h}$ and then exposed to an LED lamp for $1 \mathrm{~h}$ with a power density of $12 \mathrm{~mW} \mathrm{~cm}^{-2}$, all nanostructures exhibited obvious cytotoxicities toward A549 cells with increasing incubation concentration. To further elucidate the cell death mechanism, A549 cells were double-labeled with Annexin VFITC (fluorescein isothiocyanate) and PI (propidium iodide) before flow cytometry analysis. Compared with control groups (Fig. S17c, e, g), a clear increasing apop-
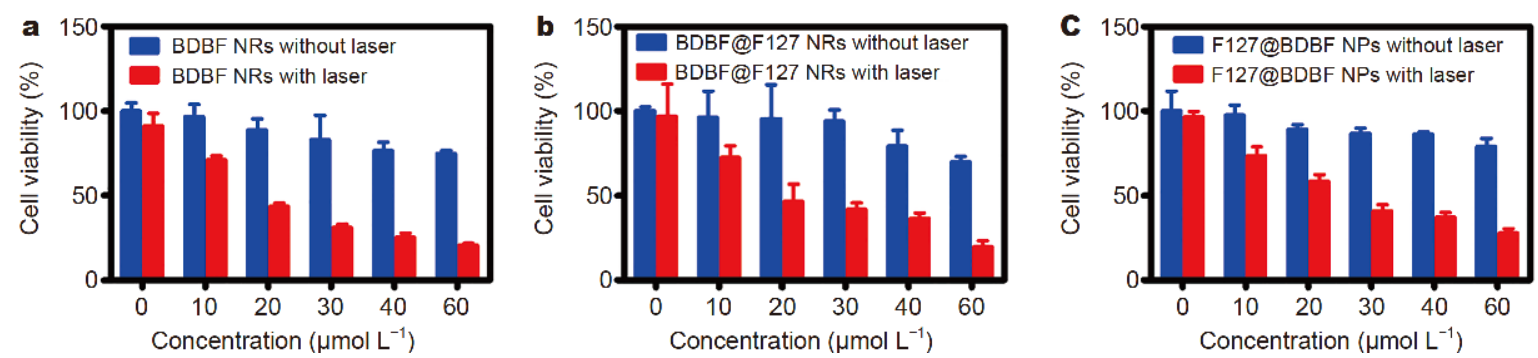

Figure 5 In vitro dark and phototoxicities of BDBF NRs (a), BDBF@F127 NRs (b), and F127@BDBF NPs (c) against A549 cells at different concentrations of BDBF $\left(0-60 \mu \mathrm{mol} \mathrm{L}^{-1}\right)$ with an LED lamp at a power density of $12 \mathrm{~mW} \mathrm{~cm}{ }^{-2}$ for $60 \mathrm{~min}$. 
totic rate $(\mathrm{Q} 2+\mathrm{Q} 3)$ was observed in the nanostructure experimental groups with $1 \mathrm{~h}$ irradiation time (Fig. S17d, $\mathrm{f}, \mathrm{h}$ ). The ratios of apoptosis cells were almost $100 \%$ due to the high concentrations of these nanostructures. The half-maximal inhibitory concentration $\left(\mathrm{IC}_{50}\right)$ towards A549 cells was also measured for various nanostructures (Table S1). Furthermore, the phototoxicities of these nanostructures against HeLa cells were measured (Fig. S18); remarkable decrease in cell viability was observed after HeLa cells were incubated with these NPs for $6 \mathrm{~h}$ and then irradiated using an LED lamp for $1 \mathrm{~h}$, indicating that these nanostructures also exhibited good PDT efficiency against HeLa cells. Trypan Blue, which was used to stain the apoptotic cells with observable blue color under an optical microscope, was used to visualize the cell apoptosis caused by PDT. As shown in Fig. S19, A549 cells were treated with the same concentration of BDBF NRs, BDBF@F127 NRs, or F127@BDBF NPs, whereas the control groups were only incubated with the medium. A slight blue color was observed in the control groups or the groups pretreated with the nanostructures without light irradiation, indicating that the nanostructures were not harmful to A549 cells in the absence of light irradiation. When A549 cells were irradiated with light, no blue color was observed for the control group, but the cells cultured with these nanostructures were killed.

Next, the in vitro therapeutic effect was visually evaluated by staining with calcein-AM and PI to identify live (green) and dead/late apoptotic (red) cells, respectively. As shown in Fig. S20, most cells incubated only with the culture medium in dark or culture medium with light irradiation showed strong green fluorescence, indicating that they were alive. The cells cultured with these nanostructures without light irradiation for $24 \mathrm{~h}$ were alive with intense green fluorescence and ignorable red fluorescence, indicating nice biocompatibility of these nanostructures. Nevertheless, much more A549 cells were found to be dead with a higher red fluorescence after incubation with F127@BDBF NPs under light irradiation in contrast with those treated with BDBF NRs and BDBF@F127 NRs, indicating that the three nanostructures inhibited the growth of cancer cells effectively.

\section{CONCLUSIONS}

In summary, three nanostructures with different morphologies based on BDBF were obtained using reprecipitation method. BDBF could self-assemble into nanorods in water, whereas BDBF@F127 NRs were prepared by modifying the nanorods with F127. Spherical
F127@BDBF NPs were also fabricated by encapsulating BDBF with F127. BDBF NRs and BDBF@F127 NRs showed identical fluorescence emission peaks with higher QYs, whereas F127@BDBF NPs exhibited different fluorescence emission with a low FQY. The three nanostructures exhibited similar ${ }^{1} \mathrm{O}_{2}$ generation capability, higher than BDBF molecule in the dissolved state, and they could be utilized for bioimaging and tumor inhibition effectively, indicating that they can be applied for anticancer PDT. The different self-assembly structures of BDBF provide a promising method to fabricate desired morphologies with high luminescence performance and efficient photosensitization for image-guided PDT.

Received 15 June 2019; accepted 22 July 2019;

published online 22 August 2019

1 Felsher DW. Cancer revoked: Oncogenes as therapeutic targets. Nat Rev Cancer, 2003, 3: 375-379

2 Osedach TP, Andrew TL, Bulović V. Effect of synthetic accessibility on the commercial viability of organic photovoltaics. Energy Environ Sci, 2013, 6: 711

3 Huang $\mathrm{P}$, Lin J, Wang X, et al. Light-triggered theranostics based on photosensitizer-conjugated carbon dots for simultaneous enhanced-fluorescence imaging and photodynamic therapy. Adv Mater, 2012, 24: 5104-5110

4 Wang $\mathrm{H}$, Xie $\mathrm{H}$, Wang J, et al. Self-assembling prodrugs by precise programming of molecular structures that contribute distinct stability, pharmacokinetics, and antitumor efficacy. Adv Funct Mater, 2015, 25: 4956-4965

5 Huang $\mathrm{Z}, \mathrm{Xu} \mathrm{H}$, Meyers $\mathrm{AD}$, et al. Photodynamic therapy for treatment of solid tumors-Potential and technical challenges. Technol Cancer Res Treat, 2008, 7: 309-320

6 Dougherty TJ, Gomer CJ, Henderson BW, et al. Photodynamic therapy. J Natl Cancer Institute, 1998, 90: 889-905

7 Wang C, Cheng L, Liu Z. Upconversion nanoparticles for photodynamic therapy and other cancer therapeutics. Theranostics, 2013, 3: 317-330

8 Zhou Z, Song J, Nie L, et al. Reactive oxygen species generating systems meeting challenges of photodynamic cancer therapy. Chem Soc Rev, 2016, 45: 6597-6626

9 Lucky SS, Soo KC, Zhang Y. Nanoparticles in photodynamic therapy. Chem Rev, 2015, 115: 1990-2042

10 Juarranz Á, Jaén P, Sanz-Rodríguez F, et al. Photodynamic therapy of cancer. Basic principles and applications. Clin Transl Oncol, 2008, 10: 148-154

11 Chang CC, Hsieh MC, Lin JC, et al. Selective photodynamic therapy based on aggregation-induced emission enhancement of fluorescent organic nanoparticles. Biomaterials, 2012, 33: 897-906

12 Pei Q, Hu X, Zheng X, et al. Light-activatable red blood cell membrane-camouflaged dimeric prodrug nanoparticles for synergistic photodynamic/chemotherapy. ACS Nano, 2018, 12: 1630-1641

13 Zheng X, Wang L, Liu S, et al. Nanoparticles of chlorin dimer with enhanced absorbance for photoacoustic imaging and phototherapy. Adv Funct Mater, 2018, 28: 1706507

14 He H, Zheng X, Liu S, et al. Diketopyrrolopyrrole-based carbon 
dots for photodynamic therapy. Nanoscale, 2018, 10: 10991-10998

15 Chen W, Ouyang J, Liu H, et al. Black phosphorus nanosheetbased drug delivery system for synergistic photodynamic/photothermal/chemotherapy of cancer. Adv Mater, 2017, 29: 1603864

16 Sun C, Ji S, Li F, et al. Diselenide-containing hyperbranched polymer with light-induced cytotoxicity. ACS Appl Mater Interfaces, 2017, 9: 12924-12929

17 Kessel D. Apoptosis, paraptosis and autophagy: Death and survival pathways associated with photodynamic therapy. Photochem Photobiol, 2019, 95: 119-125

18 Li X, Kim CY, Lee S, et al. Nanostructured phthalocyanine assemblies with protein-driven switchable photoactivities for biophotonic imaging and therapy. J Am Chem Soc, 2017, 139: 1088010886

19 Yu B, Wei H, He Q, et al. Efficient uptake of ${ }^{177}$ Lu-porphyrin-PEG nanocomplexes by tumor mitochondria for multimodal-imagingguided combination therapy. Angew Chem Int Ed, 2018, 57: 218222

20 Fan W, Lu N, Xu C, et al. Enhanced afterglow performance of persistent luminescence implants for efficient repeatable photodynamic therapy. ACS Nano, 2017, 11: 5864-5872

21 Zhao J, Xu K, Yang W, et al. The triplet excited state of bodipy: Formation, modulation and application. Chem Soc Rev, 2015, 44: 8904-8939

22 Guo Z, Zou Y, He H, et al. Bifunctional platinated nanoparticles for photoinduced tumor ablation. Adv Mater, 2016, 28: 1015510164

23 Ye S, Rao J, Qiu S, et al. Rational design of conjugated photosensitizers with controllable photoconversion for dually cooperative phototherapy. Adv Mater, 2018, 30: 1801216

24 Tang Q, Si W, Huang C, et al. An aza-bodipy photosensitizer for photoacoustic and photothermal imaging guided dual modal cancer phototherapy. J Mater Chem B, 2017, 5: 1566-1573

25 Cai Y, Liang P, Tang Q, et al. Diketopyrrolopyrrole-triphenylamine organic nanoparticles as multifunctional reagents for photoacoustic imaging-guided photodynamic/photothermal synergistic tumor therapy. ACS Nano, 2017, 11: 1054-1063

26 Singh S, Aggarwal A, Bhupathiraju NVSDK, et al. Glycosylated porphyrins, phthalocyanines, and other porphyrinoids for diagnostics and therapeutics. Chem Rev, 2015, 115: 10261-10306

27 Guo M, Mao H, Li Y, et al. Dual imaging-guided photothermal/ photodynamic therapy using micelles. Biomaterials, 2014, 35: 4656-4666

28 Li X, Lee S, Yoon J. Supramolecular photosensitizers rejuvenate photodynamic therapy. Chem Soc Rev, 2018, 47: 1174-1188

29 Shen Y, Shuhendler AJ, Ye D, et al. Two-photon excitation nanoparticles for photodynamic therapy. Chem Soc Rev, 2016, 45: 6725-6741

30 Fan W, Huang P, Chen X. Overcoming the Achilles' heel of photodynamic therapy. Chem Soc Rev, 2016, 45: 6488-6519

31 Zhang W, Lin W, Zheng X, et al. Comparing effects of redox sensitivity of organic nanoparticles to photodynamic activity. Chem Mater, 2017, 29: 1856-1863

32 Li B, Li J, Fu Y, et al. Porphyrins with four monodisperse oligofluorene arms as efficient red light-emitting materials. J Am Chem Soc, 2004, 126: 3430-3431

33 DeRosa M, Crutchley RJ. Photosensitized singlet oxygen and its applications. Coord Chem Rev, 2002, 233-234: 351-371

34 Abbas M, Zou Q, Li S, et al. Self-assembled peptide- and proteinbased nanomaterials for antitumor photodynamic and photo- thermal therapy. Adv Mater, 2017, 29: 1605021

35 Luo J, Xie Z, Lam JWY, et al. Aggregation-induced emission of 1methyl-1,2,3,4,5-pentaphenylsilole. Chem Commun, 2001, 17401741

36 Mei J, Leung NLC, Kwok RTK, et al. Aggregation-induced emission: Together we shine, united we soar! Chem Rev, 2015, 115: 11718-11940

$37 \mathrm{Wu} \mathrm{W}, \mathrm{Mao} \mathrm{D}, \mathrm{Hu}$ F, et al. A highly efficient and photostable photosensitizer with near-infrared aggregation-induced emission for image-guided photodynamic anticancer therapy. Adv Mater, 2017, 29: 1700548

38 Mao D, Wu W, Ji S, et al. Chemiluminescence-guided cancer therapy using a chemiexcited photosensitizer. Chem, 2017, 3: 9911007

39 Yuan Y, Feng G, Qin W, et al. Targeted and image-guided photodynamic cancer therapy based on organic nanoparticles with aggregation-induced emission characteristics. Chem Commun, 2014, 50: 8757-8760

40 Zhang R, Zhang CJ, Feng G, et al. Specific light-up probe with aggregation-induced emission for facile detection of chymase. Anal Chem, 2016, 88: 9111-9117

41 Gao H, Zhang X, Chen C, et al. Unity makes strength: How aggregation-induced emission luminogens advance the biomedical field. Adv Biosys, 2018, 2: 1800074

42 Jiang N, Shen T, Sun JZ, et al. Aggregation-induced emission: Right there shining. Sci China Mater, 2019, 62: 1227-1235

$43 \mathrm{Gu} \mathrm{B}, \mathrm{Wu} \mathrm{W}, \mathrm{Xu} \mathrm{G}$, et al. Precise two-photon photodynamic therapy using an efficient photosensitizer with aggregation-induced emission characteristics. Adv Mater, 2017, 29: 1701076

44 Liu B. Aggregation-induced emission: Materials and biomedical applications. MRS Bull, 2017, 42: 458-463

45 Qi J, Chen C, Zhang X, et al. Light-driven transformable optical agent with adaptive functions for boosting cancer surgery outcomes. Nat Commun, 2018, 9: 1848

46 Chen $\mathrm{C}, \mathrm{Ou} \mathrm{H}$, Liu R, et al. Regulating the photophysical property of organic/polymer optical agents for promoted cancer phototheranostics. Adv Mater, 2019, 35: 1806331

47 Ni X, Zhang X, Duan X, et al. Near-infrared afterglow luminescent aggregation-induced emission dots with ultrahigh tumor-to-liver signal ratio for promoted image-guided cancer surgery. Nano Lett, 2018, 19: 318-330

48 Xu M, Wang X, Wang Q, et al. Analyte-responsive fluorescent probes with AIE characteristic based on the change of covalent bond. Sci China Mater, 2019, 62: 1236-1250

49 Lovell JF, Liu TWB, Chen J, et al. Activatable photosensitizers for imaging and therapy. Chem Rev, 2010, 110: 2839-2857

50 Yogo T, Urano Y, Ishitsuka Y, et al. Highly efficient and photostable photosensitizer based on bodipy chromophore. J Am Chem Soc, 2005, 127: 12162-12163

51 McDonnell SO, Hall MJ, Allen LT, et al. Supramolecular photonic therapeutic agents. J Am Chem Soc, 2005, 127: 16360-16361

52 Xu S, Yuan Y, Cai X, et al. Tuning the singlet-triplet energy gap: A unique approach to efficient photosensitizers with aggregationinduced emission (AIE) characteristics. Chem Sci, 2015, 6: 58245830

$53 \mathrm{Xu} \mathrm{S}, \mathrm{Wu} \mathrm{W}$, Cai X, et al. Highly efficient photosensitizers with aggregation-induced emission characteristics obtained through precise molecular design. Chem Commun, 2017, 53: 8727-8730

54 Yuan Y, Xu S, Zhang CJ, et al. Dual-targeted activatable photosensitizers with aggregation-induced emission (AIE) characteristics 
for image-guided photodynamic cancer cell ablation. J Mater Chem B, 2016, 4: 169-176

$55 \mathrm{Wu}$ W, Feng G, Xu S, et al. A photostable far-red/near-infrared conjugated polymer photosensitizer with aggregation-induced emission for image-guided cancer cell ablation. Macromolecules, 2016, 49: 5017-5025

$56 \mathrm{Wu}$ W, Xu S, Qi G, et al. A cross-linked conjugated polymer photosensitizer enables efficient sunlight-induced photooxidation. Angew Chem Int Ed, 2019, 58: 3062-3066

57 Liu S, Zhang H, Li Y, et al. Strategies to enhance the photosensitization: Polymerization and the donor-acceptor even-odd effect. Angew Chem Int Ed, 2018, 57: 15189-15193

58 Wang S, Wu W, Manghnani P, et al. Polymerization-enhanced two-photon photosensitization for precise photodynamic therapy. ACS Nano, 2019, 13: 3095-3105

$59 \mathrm{Wu}$ W, Mao D, Xu S, et al. Polymerization-enhanced photosensitization. Chem, 2018, 4: 1937-1951

60 Qi J, Chen C, Ding D, et al. Aggregation-induced emission luminogens: Union is strength, gathering illuminates healthcare. Adv Healthcare Mater, 2018, 7: 1800477

$61 \mathrm{Yu} \mathrm{CYY}, \mathrm{Xu} \mathrm{H}$, Ji S, et al. Mitochondrion-anchoring photosensitizer with aggregation-induced emission characteristics synergistically boosts the radiosensitivity of cancer cells to ionizing radiation. Adv Mater, 2017, 29: 1606167

62 Gao Y, Zheng QC, Xu S, et al. Theranostic nanodots with aggregation-induced emission characteristic for targeted and imageguided photodynamic therapy of hepatocellular carcinoma. Theranostics, 2019, 9: 1264-1279

63 Cai X, Mao D, Wang C, et al. Multifunctional liposome: A bright AIEgen-lipid conjugate with strong photosensitization. Angew Chem Int Ed, 2018, 57: 16396-16400

64 Feng G, Wu W, Xu S, et al. Far red/near-infrared AIE dots for image-guided photodynamic cancer cell ablation. ACS Appl Mater Interfaces, 2016, 8: 21193-21200

65 Fateminia SMA, Kacenauskaite L, Zhang CJ, et al. Simultaneous increase in brightness and singlet oxygen generation of an organic photosensitizer by nanocrystallization. Small, 2018, 14: 1803325

66 Wang J, Mao W, Lock LL, et al. The role of micelle size in tumor accumulation, penetration, and treatment. ACS Nano, 2015, 9: 7195-7206

67 Feng G, Mao D, Liu J, et al. Polymeric nanorods with aggregationinduced emission characteristics for enhanced cancer targeting and imaging. Nanoscale, 2018, 10: 5869-5874

68 Cai Y, Si W, Tang Q, et al. Small-molecule diketopyrrolopyrrolebased therapeutic nanoparticles for photoacoustic imaging-guided photothermal therapy. Nano Res, 2016, 10: 794-801

69 Liang P, Huang X, Wang Y, et al. Tumor-microenvironment-re- sponsive nanoconjugate for synergistic antivascular activity and phototherapy. ACS Nano, 2018, 12: 11446-11457

70 Liang P, Wang Y, Wang P, et al. Triphenylamine flanked furandiketopyrrolopyrrole for multi-imaging guided photothermal/ photodynamic cancer therapy. Nanoscale, 2017, 9: 18890-18896

71 Zhang J, Xu B, Tian W, et al. Tailoring the morphology of AIEgen fluorescent nanoparticles for optimal cellular uptake and imaging efficacy. Chem Sci, 2018, 9: 2620-2627

72 Han X, Bai Q, Yao L, et al. Highly efficient solid-state near-infrared emitting material based on triphenylamine and diphenylfumaronitrile with an EQE of $2.58 \%$ in nondoped organic light-emitting diode. Adv Funct Mater, 2015, 25: 7521-7529

73 Wang Y, Han X, Xi W, et al. Bright AIE nanoparticles with F127 encapsulation for deep-tissue three-photon intravital brain angiography. Adv Healthcare Mater, 2017, 6: 1700685

74 Liu W, Wang Y, Han X, et al. Fluorescence resonance energy transfer (FRET) based nanoparticles composed of AIE luminogens and NIR dyes with enhanced three-photon near-infrared emission for in vivo brain angiography. Nanoscale, 2018, 10: 10025-10032

75 Yu J, Zhang X, Hao X, et al. Near-infrared fluorescence imaging using organic dye nanoparticles. Biomaterials, 2014, 35: 3356-3364

76 Hao X, Zhou M, Zhang X, et al. Highly luminescent and photostable core-shell dye nanoparticles for high efficiency bioimaging. Chem Commun, 2014, 50: 737-739

77 Zheng X, Wang L, Pei Q, et al. Metal-organic framework@porous organic polymer nanocomposite for photodynamic therapy. Chem Mater, 2017, 29: 2374-2381

Acknowledgements This work was financially supported by the National Natural Science Foundation of China (21835001, 51773080, 21674041, 51573068, 21221063, and 81870117), the Program for Changbaishan Scholars of Jilin Province, Jilin Province project (20160101305JC), Jilin Province Science and Technology Development Plan (20190201252JC), and "Talents Cultivation Program" of Jilin University.

Author contributions Han W designed and performed the experiments, analyzed data and wrote the paper; Zhang S, Deng R, Qian J and Zheng $\mathrm{X}$ performed partial experiments. Yan $\mathrm{F}$ designed partial experiments. Tian $\mathrm{W}$ conceived the framework of this paper and revised the paper. All authors contributed to the general discussion.

Conflict of interest The authors declare that they have no conflict of interest.

Supplementary information Supporting data are available in the online version of the paper. 


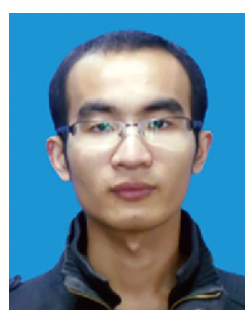

Wenkun Han received his BSc degree from Jilin University (JLU) in 2015. He is currently a PhD candidate under the supervision of Prof. Wenjing Tian. His research interest focuses on the selfassembly fluorogens for bioimaging and anticancer applications.

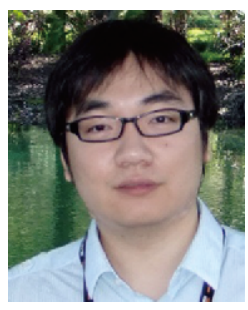

Fei Yan is an associate professor in the Chemistry Department, JLU. He received his $\mathrm{PhD}$ degree from JLU in 2010, and worked as a postdoctoral fellow first at Ohio State University and then at the University of Minnesota during 2010-2017. His research interests include the bioinorganic chemistry, translational nanomedicine and cancer epigenetics.

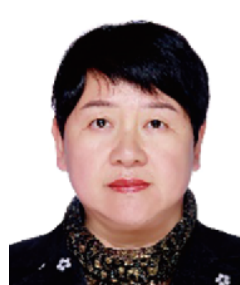

Wenjing Tian received her Bachelor's degree from the Department of Physics, JLU in 1988, and her $\mathrm{PhD}$ from Changchun Institute of Applied Chemistry, Chinese Academy of Sciences in 1993. She was a postdoctoral fellow at the Department of Chemistry, JLU from 1994 to 1996. She is currently a professor at the State Key Laboratory of Supramolecular Structure and Materials of JLU. Her research interest focuses on organic/polymer optoelectronic materials and devices.

\section{具有自组装纳米结构的AIE光敏剂用于增强光动} 力治疗

韩文坤 ${ }^{1}$, 张松 ${ }^{1}$, 邓荣 ${ }^{2}$, 杜阳阳 ${ }^{2}$, 钱靖宇 ${ }^{1}$, 郑孝华 ${ }^{3}$, 徐斌 ${ }^{1}$, 谢志刚 ${ }^{3}$, 间飞 ${ }^{2^{*}}$, 田文晶 ${ }^{1^{*}}$

摘要 基于具有聚集诱导发光(AIE) 性质的 2,3-双 (4' - (二苯基)[1, $1^{\prime}$-联苯]-4-基]富甲腈(BDBF)分子, 制备了三种纳米结构并用于 图像引导光动力学治疗 $(\mathrm{PDT})$. 普兰尼克F127可包封 BDBF形成常 见的球形纳米粒子(F127@BDBF NPs), 该纳米粒子可发射红色苂 光, 苂光量子效率(FQY)为 $9.8 \%$. 此外, $\mathrm{BDBF}$ 也可在水中自组装成 纳米棒(BDBF NRs). 与F127@BDBF NPs相比, BDBF NRs呈现出较 强的橙色苂光, 具有较高的苂光量子产率 (23.3\%), 以及基本相同的 单线态氧 $\left({ }^{1} \mathrm{O}_{2}\right)$ 产生能力. 利用F127对BDBF NRs进行进一步修饰可 得到BDBF@F127 NRs, 该纳米粒子仍然保持了棒状形貌和较好 的 ${ }^{1} \mathrm{O}_{2}$ 产生能力. 同时, 与溶解态的 BDBF相比, 三种纳米结构的单 线态氧产生效率增强. 这些纳米结构在水溶液和生理条件下具有 良好的稳定性. 细胞的光毒性实验表明, 三种纳米结构均能有效抑 制肿瘤细胞增殖. 因此, 通过简单的自组装方法制备高苂光量子效 率和较强单线态氧产生能力的纳米结构可作为一种有效的途径来 增强光动力. 